\title{
PRIMARY YOLK SAC TUMOUR OF LIVER WITH UNUSUAL CLINICAL PRESENTATION MIMICKING ACUTE APPENDICITIS
}

\author{
${ }^{1}$ Hosala M, ${ }^{1}$ Laca L, ${ }^{2}$ Hosalova Matisova J, ${ }^{1}$ Janik J, ${ }^{1}$ Adamik M, ${ }^{1}$ Palkoci B, ${ }^{3}$ Kajo K. \\ ${ }^{1}$ Clinic of Transplant and Vascular Surgery, Jessenius Faculty of Medicine in Martin, Comenius University and \\ University Hospital in Martin, ${ }^{2}$ Clinic of Anesthesiology and Intensive Medicine, Jessenius Faculty of Medicine in \\ Martin, Comenius University and University Hospital in Martin, ${ }^{3}$ Department of Pathology of Slovak Medical \\ University and Oncologic Institute of St. Elizabeth, Bratislava, Slovak Republic
}

\begin{abstract}
A b s t r a c t
Primary hepatic yolk sac tumour (YST) is an extremely rare tumour of the liver. The exact etiology of primary hepatic YST is still unclear. This tumour is very aggressive with ability to spread into distant sites, where it can lead to the first clinical presentation. The authors present a case of a 20 -year-old woman with fever and pain in the epigastrium, which later on moved to the right hypogastrium clinically resembling acute appendicitis. Ultrasound revealed tumorous mass in the liver and hypoechogenous tumorous mass in the area of right adnexes and terminal ileum. Tumorous mass in the liver was confirmed on the CT scan. Markedly raised levels of serum $\alpha$-fetoprotein concentration were detected. Laparoscopic appendectomy was performed and there was bioptically verified a diagnosis of YST. Afterwards, patient underwent right hemihepatectomy, right hemicolectomy and right adnexectomy. There was found no evidence of the gonadal, mediastinal, retroperitoneal and central nervous system origin of the YST. Histological findings from the liver proved primary yolk sac tumour of the liver with the metastatic spread into the appendix, caecum and regional lymph nodes.
\end{abstract}

Key words: liver neoplasm - yolk sac tumour - endodermal sinus tumour - alpha-fetoprotein

\section{INTRODUCTION}

Yolk sac tumour (or endodermal sinus tumour) is a very rare germ cell tumour, which usually arise from the ovary or testis, although there have been reports of such tumours arising in extragonadal sites. Extragonadal yolk sac tumour is a rare malignancy; which occurs in approximately $20 \%$ of cases from all yolk sac tumours. Its main distribution is along the midline of the body at three principal sites: mediastinum, central nervous system and retroperitoneum. The most common extragonadal sites are sacrococcygeal (78\%), mediastinal, intracranial (pineal gland: 62\%; suprasellar region: 31\%; both areas: $7 \%$ ) and retroperitoneal. Other locations include the pelvis, cervix or uterus, vagina, prostate, abdominal wall, bile duct, omentum, kidney, hernia sac, stomach, nasal cavity, lip and neck $[1,2,3,4,5,6]$. Primary yolk sac tumour of the liver was first time reported in 1975 by Hart in an 18-month-old boy who died 6.5 months after an extended hemihepatectomy [7]. Since then a further 17 cases have been reported.

The etiopathogenesis of the extragonadal yolk sac tumours is still controversial. One hypothesis suggests these tumours may arise from misplaced or arrested germ cells during embryogenesis [8], while others have proposed these tumours arise from aberrant differentiation of somatic cells [9].

\section{CASE REPORT}

A 20-year-old woman was admitted to our department with 3 days lasting pain in the epigastrium and with fever lasting for one week. Her medical history was unremarkable. Her

Address for correspondence:

MUDr. Michal Hosala, PhD., Clinic of Transplant and Vascular Surgery, Jessenius Medical Faculty, Comenius University and University Hospital, Kollarova Str. N. 2, 03659 Martin, Slovakia

Phone: +421 434203 445; e-mail: hosalamichal@gmail.com 
only regular medication was the combined oral contraceptive pill. On admission, physical examination revealed pain in periumbilical area and epigastrium during palpation and percussion, which later moved to the right hypogastrium. It also showed abdominal distension and swollen lymph nodes in both groins, but without signs of peritoneal irritation. On the ultrasound of the abdomen there was found hypoechogenous tumorous mass sized $4.4 \mathrm{x}$ 2.6 in the area of right adnexes and terminal ileum and also nonhomogenous structure sized $7.9 \times 8.7 \mathrm{~cm}$ in the right lobe of the liver. Subsequently, the CT scan was done, which revealed big tumorous mass in the right lobe of the liver sized AP x LL CC $15 \times 12 \times 15 \mathrm{~cm}$ and tumorous mass on the caecum sized $2.8 \times 3.9 \mathrm{~cm}$ (Fig. 1, 2). The laboratory tests showed an increased white blood cell count to $14.6 \times 10^{9} / \mathrm{L}$ (reference: $4-10 \times 10^{9} / \mathrm{L}$ ) and CRP was $133 \mathrm{mg} / \mathrm{L}$ (reference: < $5 \mathrm{mg} / \mathrm{L}$ ). Laparoscopic exploration was performed with findings of big tumour localized in the right lobe of the liver and phlegmonously changed appendix. In the first phase laparoscopic appendectomy was done and biopsy from the liver tumour was taken. The most probable diagnosis was considered hepatocellular carcinoma. In serum was detected extremely high level of $\alpha$-fetoprotein (AFP) $>71753-100000 \mathrm{ng} / \mathrm{mL}$ (reference: < $20 \mathrm{ng} / \mathrm{mL}$ ), The carbohydrate antigen (CA) 19-9, CA 125, CEA, the human chorionic gonadotropin level, blood amylase, aspartate transferase (AST), alanine transferase (ALT), Quick time and bilirubin levels were within the normal ranges. Serology testing was negative for viral hepatitis. Later on, histological samples from appendix proved the diagnosis of yolk sac tumour. According to the results of histology, right hemihepatectomy, right hemicolectomy and right adnexectomy were performed.

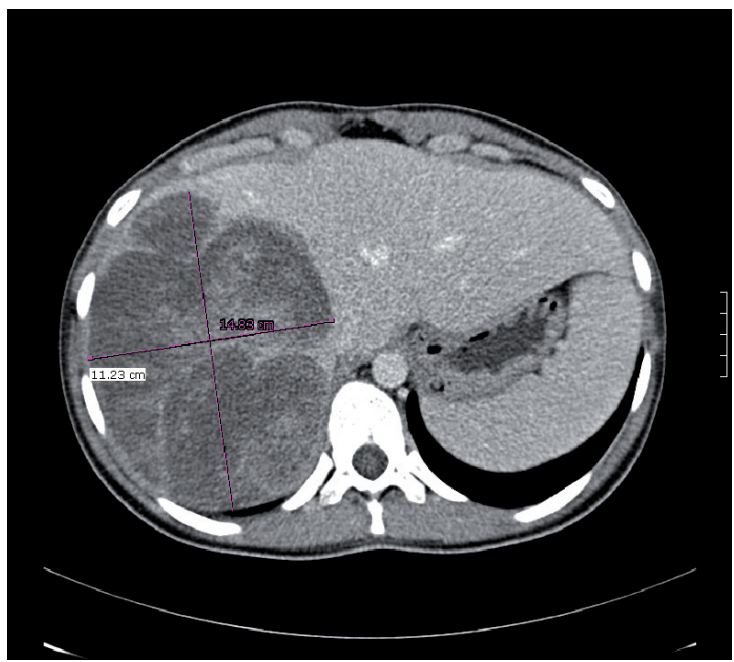

Fig. 1. Primary yolk sac tumour of the liver in 20-yearold woman.

Axial CT scan enhanced with contrast shows a welldefined solid mass of the right lobe of the liver

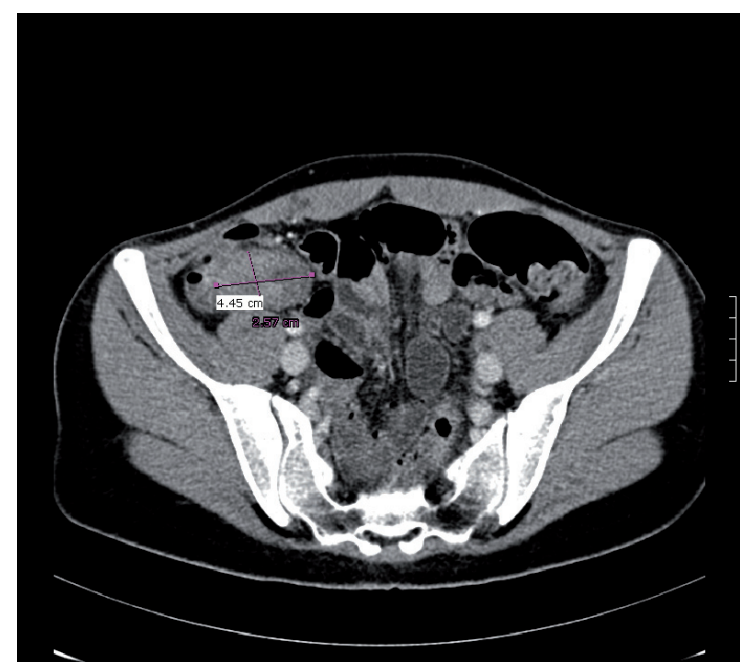

Fig.2. Metastasis of YST to the caecum

Axial CT scan shows the metastasis of the tumour to the caecum

Sectioning of the right lobe of the liver revealed a tumorous mass infiltrating capsule (about $15 \mathrm{~cm}$ in diameter) (Fig. 3). On the resected part of the large intestine was found exophytically growing tumour with $2.5-3 \mathrm{~cm}$ in diameter. In the resected part of mesocolon were 17 lymph nodes. In 3 of them were present metastases. Tumorous mass has soft consistency, mixed solid and cystic character, with areas of haemorrhage and necrosis. Right adnexa looked macroscopically normal. 


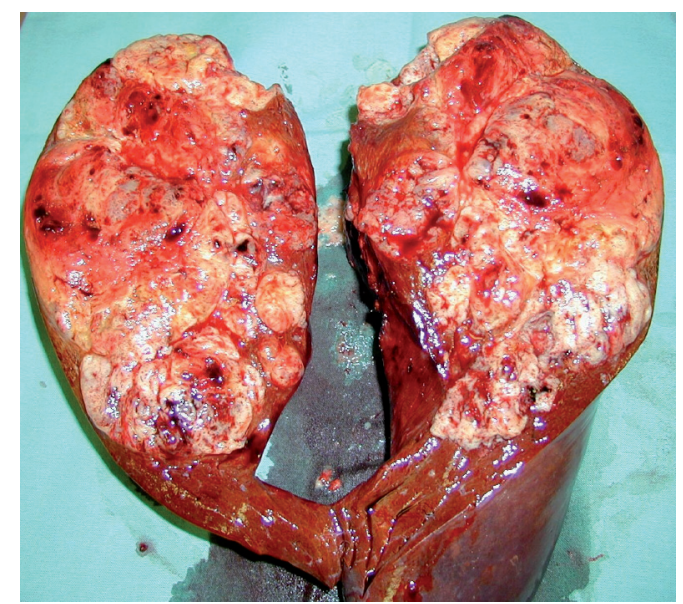

Fig.3. Sectioning of the right lobe of the liver

Sectioning of the right lobe of the liver revealed a tumorous mass infiltrating capsule (about $15 \mathrm{~cm}$ in diameter)

Microscopically, the tumours were characterized by almost identical histomorphologic characteristics and in its image contained a variety of somatic and extraembryonic endodermal differentiation. There were present districts with solid, glandular, hepatoid, papillary, intestinal and polyvesical growth patterns. The most characteristic feature of tumour was reticular growth pattern with sparse oedematous stroma, with typical cytoplasmic PASpositive and diastasis-resistant hyaline globules, as well as pathognomic papillary formations with a central vessels (Schiller-Duval Bodies) (Fig. 4). Tumorous cells were characterized by high mitotic activity. Tumorous cells were imunohistochemically positive for CK8/18, AFP, CEA, Vim (+/-), PLAP (+/-) and negative for hCG, CD30 and hepar1. On the right ovarium were found cystic changes, but without signs of tumorous infiltration.



Fig.4. Histological features of yolk sac tumour with pathognomic Schiller-Duval Bodies (in circles). H\&E, magnification $\mathrm{x} 200$

In early postoperative period were not present any complications. The patient was discharged in good condition and a month after the operation received standard adjuvant chemotherapy with the BEP regimen (bleomycin, etoposide and cisplatin). A total of four 
cycles of systemic chemotherapy were performed. Patient was admitted with signs of intestinal sub-occlusion 15 months after second operation. Explorative laparotomy revealed numerous small intestinal adhesions and the adhesiolysis was performed. No other complications were present and relapse was not seen during 3, 6, 9, 12, 24, 36 and 48-month follow ups (Fig. 5).



Fig.5. CT scan of abdomen 6 months after operation

Axial CT scan of abdomen reveals regeneration of the liver parenchyma 6 months after operation

\section{DISCUSSION}

Yolk sac tumour accounts for less than 1\% of germ cell tumours [10]. An extragonadal location of YST is very rare and the tumour is usually localized in the mediastinum, pineal gland, sacrococcygeal region and vagina [11]. Primary hepatic YST usually arise in children or young adults [12.13]. Only 17 cases of primary hepatic YST were described in literature so far. This tumour is very aggressive with ability to spread into distant sites, where it can lead to the first clinical presentation. To our knowledge, there has not yet been reported any case of primary yolk sac tumour, with first symptomatology arising from the metastatic affection of the caecum and appendix, which had resembling acute appendicitis.

In differential diagnoses of hepatic YST are considered hepatocellular carcinoma and hepatoblastoma, which are also presented by increased levels of serum $\alpha$-fetoprotein [14]. Diagnosis of a yolk-sac tumour can be made by taking a biopsy during surgery and freezing sections, which can then be pathologically assessed. A skilled pathologist should be used for such assessment, especially for mixed germ-cell tumours. There is required very close cooperation between surgeon, pathologist and oncologist.

Regular follow ups of our patient were performed. There was found no evidence of tumour recurrence even 4 years after surgery. Since YST is a neoplasm with an unpredictable biological behaviour, we will continue with close clinical follow up. There is a need to establish long term outcome of surgically treated primary yolk sac tumours of the liver.

In conclusion, primary yolk sac tumour of the liver, even if it is a rare malignancy, should always be considered as an alternative diagnosis to hepatocellular carcinoma in a young patient with highly elevated serum $\alpha$-fetoprotein concentrations and a tumour within a noncirrhotic liver. 


\section{REFERENCES}

1. Hart WR. Primary endodermal sinus (yolk sac) tumour of the liver. First reported case. Cancer 1975;35:1453-8.

2. Pizzo PA, Poplack DG (Eds): Principles and practice of pediatric oncology, third edition. Lippincott-Raven, Philadelphia, 1997.

3. Dede M, Pabuccu R, Yagci G, Yenen MC, Goktolga U, Gunhan O. Extragonadal yolk sac tumor in pelvic localization. A case report and literature review. Gynecol Oncol. 2004;92:989-991.

4. Morinaga S, Nishiya H, Inafuku T. Yolk sac tumor of the liver combined with hepatocellular carcinoma. Arch Pathol Lab Med. 1996;120:687-690.

5. Wong, N.A.C.S., DęCosta, H., Barry, R.E., Alderson, D., Moorghen, M.: Primary yolk sac tumor of the liver in adulthood. J. Clin. Pathol 1998;51:939-940.

6. Razzi S, Luisi S, Gabbanini M, Lazzeri L, Mazzini M, Petraglia F. Yolk sac tumor in a young girl: a case report. Gynecol Endocrinol. 2005;20:334-335.

7. Zhang B., Gao S., Chen Y., Wu Y. Primary Yolk Sac Tumor in Pancreas Showing Liver Metastases: a case report. Korean J Radiol. 11(4), Jul/Aug 2010.

8. Mokhallagh Sani P., Tarzamni M.K., Aslanabadi S., Eftekhar Sadat A.T., Mokhallagh Sani A., Nezami N. Pure Yolk Sac (Endodermal Sinus)Tumor of the Liver: A Case Report, Iran J Radiol. 2009, 6(2)

9. Deshmukh, C., Bakshi, A., Bhagwat, R., Kurkure, P.: Yolk sac tumor of vagina. Indian J. Pediatr. 2005;72:48-49.

10. Multi, T., Khan, M.S., Muzher-U-Dua, Waqar, F.: Malignant sacrococcygeal yolk sac (endodermal sinus) tumor. J. Ayub. Med. Coll. Abbottabad. 2002;14:28-30.

11. Hong, R, Suh, C.H., Lee, M.J.: Adenocarcinoma with yolk sac tumor of the stomach: Case report with review of the literature and an immunohistochemical study. Korean J. Pathol. 2007; 41:352-357.

12. Filho, B.C., McHugh, J.B., Carrau, R.L., Kassam, A.B.: Yolk sac tumor in the nasal cavity. Am. J. Otolaryngol. 2008;29:250-254.

13. Kim, S.W., Park, J.H., Lim, M.C., Park, J.Y., Yoo, C.W., Park, S.Y.: Primary yolk sac tumor of the omentum: a case report and review of the literature. Arch. Gynecol. Obstetr. 2008;279:189-192.

14. Kumar, V., Vaiphei, K.: Intrarenal pure yolk sac tumor. Int. J. Surg. Pathol. 2007;15:204-206.

Received: October,1, 2013

Accepted: December,10, 2013 